\title{
Retrospective Analysis
}

\section{Methylprednisolone Dosage in Lumbar Epidural Steroid Injections: A Retrospective Analysis of Efficacy in Pain RELIEF}

\author{
Nadia Naushin Zaman, DO' ${ }^{1}$ Calvin Rong Chen, $\mathrm{DO}^{1}$, Ketan Patel, $\mathrm{MD}^{2}$, Tungming Leung, $\mathrm{PhD}^{3}$, and \\ Shaheda Amin Quraishi, MD4
}

Background: Epidural injections have been used for pain relief since the 1880 s. Corticosteroids are antiinflammatory medications that can alleviate pain, but also have harmful systemic adverse effects. Literature regarding methylprednisolone dosage efficacy is limited.

Objectives: To determine the lowest effective dose of methylprednisolone in a lumbar epidural steroid injection (LESI) for maximal pain relief without exposing patients to adverse events caused by steroid use.

Study Design: Retrospective chart review.

Setting: Outpatient interventional pain clinic at an academic center.

Methods: Adults ( $n=133$ ), aged 18 to 85 years, with low-back pain and radicular symptoms treated with LESI from August 2011 to November 2015. Patients who received prior cervical epidural steroid injections were excluded. Interventions were

LESI with methylprednisolone $40 \mathrm{mg}, 80 \mathrm{mg}$, or $120 \mathrm{mg}$. Main outcome measures showed change in pain score using a numeric pain scale (NPS; 0 = no pain, $10=$ excruciating pain), and patient's self-reported reduction in pain (percentage), pre- and postprocedure. The primary endpoint measurement was 2 weeks postinjection. Adverse effects were recorded.

Results: The number of patients who received each dose varied: $n=88$ received $120 \mathrm{mg}, \mathrm{n}=30$ received $80 \mathrm{mg}$, and $n=13$ received $40 \mathrm{mg}$. The NPS pain scores pre- and postprocedure for 120 mg were $8.89 \pm 1.32$ and $4.08 \pm 3.74$, (mean \pm standard deviation), respectively; for $80 \mathrm{mg}: 9.06$ \pm 1.00 and $3.75 \pm 4.00$; and for $40 \mathrm{mg}: 9.00 \pm$ 1.00 and $4.00 \pm 0.00$. Percentage of pain relief for $120 \mathrm{mg}, 80 \mathrm{mg}$, and $40 \mathrm{mg}$ was $57.26 \%, 50.74 \%$, and 57.26 , respectively $(P=0.3347) . \mathrm{n}=4 \mathrm{ex}$ perienced adverse effects, all received $120 \mathrm{mg}$. Conclusions: All 3 dosage groups had similar efficacy in pain relief, but only patients who received $120 \mathrm{mg}$ experienced adverse effects. This demonstrates that lower dosages can be used for pain relief with less potential harm to the patient.

Key words: Methylprednisolone, interlaminar epidural, radiculopathy

From : 'Department of Physical Medicine and Rehabilitation, Donald and Barbara Zucker School of Medicine at Hofstra-Northwell, Manhasset, NY; ${ }^{2}$ Department of Physical Medicine and Rehabilitation, Nassau University Medical Center, East Meadow, NY; ${ }^{3}$ Department of Biostatistics, Northwell Health, Manhasset, NY; ${ }^{4}$ Department of Neurosurgery, Northwell Health Neuroscience Institute, Great Neck, NY

Author for correspondence: Calvin Rong Chen, DO

Address: Department of Physical Medicine and Rehabilitation, Donald and Barbara Zucker School of Medicine at Hofstra-Northwell, Manhasset, NY E-mail: cchen00723@gmail.com

Disclaimer: There was no external funding in the preparation of this manuscript. Conflict of interest: Each author certifies that he or she, or a member of his or her immediate family, has no commercial association (i.e., consultancies, stock ownership, equity interest, patent/licensing arrangements, etc.) that might pose a conflict of interest in connection with the submitted manuscript. 
Back pain is the fifth most common reason individuals seek medical care (1). From 1996 to 2013, health care spending for low-back pain in the United States was estimated to be approximately $\$ 87.6$ billion $(2,3)$. Radicular back pain often carries a worse prognosis, along with disability, chronicity, poorer quality of life, and persistent pain (4). Epidural steroid injections (ESIs) are among the most widely used treatments for back pain, with the first documented injections used to treat radicular pain performed by French physicians Dr. Jean-Anthanase Sicard and Dr. Ferdinand Cathelin $(5,6)$.

Today, there are 3 main types of ESIs: interlaminar, transforaminal, and caudal. The clinical effectiveness and usefulness of epidural injections has been both widely studied and debated; however, differences in the procedural technique, medications used, and diagnostic criteria have made comparisons difficult. The most widely used epidural injection today is the interlaminar approach, which involves midline insertion of a spinal needle between the spinous processes of 2 contiguous vertebrae (7). Numerous studies on the interlaminar approach have reviewed the advantages of its contrast medium patterns under fluoroscopy, which shows excellent spread of contrast medium into the nerve root (8). The antiinflammatory effects of corticosteroids aim to relieve radicular symptoms as they travel along the nerve root by either inhibiting the synthesis or cellular release of proinflammatory mediators, and by facilitating a reversible local anesthetic effect at the injected site (9).

Various types of corticosteroids are used for ESIs. Although all have a similar mechanism of action, antiinflammatory properties, and adverse effects, the specific corticosteroid used is often based on physician preference. Methylprednisolone acetate is a particulate corticosteroid sold under the brand names Depo-Medrol and Solu-Medrol (Methylprednisolone Acetate Injectable Solution USP, $80 \mathrm{mg} /$ $\mathrm{mL}$, Teva Pharmaceuticals USA Inc., North Wales, PA) (Methylprednisolone Sodium Succinate, 40mg. $\mathrm{mL}$, Pharmacia \& Upjohn, Kalamazoo, MI). Doses of methylprednisolone acetate vary according to practice and procedure; the most commonly used doses for interlaminar ESIs are $40 \mathrm{mg}, 80 \mathrm{mg}$, and $120 \mathrm{mg}$.

With increasing use of corticosteroids in interven- tional pain procedures, elevated risk of adverse effects occurs. Serious systemic side effects of steroids range from hyperglycemia and hypertension to long-term effects, including osteoporosis and suppression of the hypothalamic-pituitary axis (HPA) (10). Less severe but more common side effects include facial flushing, headache, or pain at the injection site (11). Although ESIs have been proven to be efficacious in the treatment and management of lumbar radicular pain, evidence for an appropriate standard of corticosteroids dosing is lacking. The aim of this retrospective study was to assess the safety and efficacy of different doses of methylprednisolone for the treatment of lumbar radicular pain.

\section{METHODS}

\section{Patients}

A retrospective chart review was performed for patients who were treated by a board-certified pain management specialist in an outpatient interventional pain clinic of an academic medical center from August 2011 to November 2015. All patients were seen and treated by the same pain management specialist. This study was approved by the Northwell Health institutional review board. Inclusion criteria were adults aged 18 to 85 years, with low-back pain with radicular symptoms owing to a herniated disc disease or spinal stenosis, who received interlaminar lumbar epidural steroid injection (LESI). Exclusion criteria were any prior cervical ESIs, or any dosage given other than the dosage groups being studied. Dosage groups studied were $120 \mathrm{mg}, 80 \mathrm{mg}$, or $40 \mathrm{mg}$ of methylprednisolone. All patients received methylprednisolone manufactured by the same company (Methylprednisolone Acetate Injectable Solution USP, $80 \mathrm{mg} / \mathrm{mL}$, Teva Pharmaceuticals USA Inc., North Wales, PA). The patient was transported to the operating room, placed in the prone position, and monitored noninvasively with vital signs also monitored. The patient's back was prepped 3 times with Betadine (Avrio Health L.P., Stamford, CT) and draped in meticulous sterile fashion. The lumbar interspace was identified fluoroscopically, and the skin overlying the desired level was infiltrated with 5 $\mathrm{mL}$ of $1 \%$ lidocaine using a 25 -gauge 1 -inch needle. The epidural space was approached and entered at this level with a 20-gauge Tuohy needle by a loss of resistance technique. Following loss of resistance to 
air, aspiration was negative for cerebrospinal fluid or blood. Then, $2 \mathrm{~mL}$ of Omnipaque 300 (GE Healthcare, Chicago, IL was injected, and the epidurogram revealed spread from the desired intervertebral level space into the posterior epidural space bilaterally with no distinct cutoff. Methylprednisolone was then injected with $1 \mathrm{~mL}$ of preservative-free $1 \%$ lidocaine. The needle tract was flushed with $2 \mathrm{~mL}$ of $1 \%$ lidocaine, and the needle was removed. A sterile bandage was applied. If a patient received a second LESI, the results were also examined. Adverse effects were noted for all injections.

\section{Outcome Variables}

The primary outcome measure of efficacy was the change in pain from baseline to 2 weeks postinjection as indicated by numeric pain scale (NPS, $0=$ no pain, $10=$ excruciating pain) and patient's self-reported reduction in pain (percentage). Both the absolute change and percentage change were recorded between the dosage groups when data were available. Pain relief was defined as at least a $50 \%$ reduction in pain after the first procedure. Any adverse effects that were described by the patient were noted for both injections.

\section{Statistical Methods}

Descriptive statistics (frequency distribution for categorical variables and mean, median, standard deviation, maximum, and minimum for continuous variables) were calculated. The Mann-Whitney nonparametric test was performed to test if there was a difference in the ages between the pain improvement outcomes (< $50 \%$ vs. $\geq 50 \%$ pain NPS score reduction). The test was also used to determine if there was any difference in percentage relief after the first injection between the dosage groups, gender, and history of back surgery. The Kruskal-Wallis test was used to determine if there was a difference in age of patients among dosage groups (40 mg vs. $80 \mathrm{mg}$ vs. $120 \mathrm{mg}$ ) or differences in percentage relief after first injection by laterality and level of injection. Box plots were used to visualize the average difference of the factors analyzed by the Mann-Whitney or the Kruskal-Wallis tests. The chi-square test or the Fisher exact test was performed to test the equality of proportions between the $80 \mathrm{mg}$ and $120 \mathrm{mg}$ groups if $\geq 50 \%$ pain improvement was met and if a second injection was requested. The chi-square test or the
Fisher exact test was also used to test the association between whether the patients reached a $50 \%$ pain improvement, and gender, history of back surgery, laterality of injection, or level of injection, and between whether the patients received a second injection. The Spearman correlation analysis was used to test if there was a correlation between age and the percentage change in pain score in the patients.

\section{RESULTS}

A total of 133 patient records were reviewed in this study: 10 patients who previously underwent a cervical interlaminar steroid injection were excluded from the study. Among the 123 eligible patients whose records were analyzed, the mean age was $69 \pm 14.2$ years, and $55 \%$ were women. Most $(94 \%)$ of the patients did not have a prior surgical procedure for the lumbar spine to alleviate their pain. The distribution of the laterality of the injections is reported in Table 1. The distribution for the vertebral level of injection is reported in Table 2.

\section{Primary Outcome}

A total of 9 patients received $40 \mathrm{mg}, 28$ patients received $80 \mathrm{mg}$, and 86 patients received $120 \mathrm{mg}$ of methylprednisolone for their first LESI. The overall mean percent reduction in pain as reported by the patients after the first injection was 55\% $\pm 31.1 \%$. The absolute number change in NPS pre- and postprocedure is reported in Table 3 . The mean age and mean percent reduction of pain among the 3 dosage groups is reported in Table 4; there was a marginally significant difference in the mean age among the 3 dosage groups $(P=0.046)$. The Fisher exact test showed that there was no association between the 3 dosage groups and gender $(P=0.146)$. This indicates that the population of the dosage groups was nearly, but not entirely, homogeneous in terms of age distribution. There was no association between the 3 dosage groups regarding the groups' back pain histories, laterality of injection, or spinal levels of injection.

One patient in the $120 \mathrm{mg}$ group and one patient in the $80 \mathrm{mg}$ group did not follow-up after the procedure, so they were excluded from the final analysis due to missing data. The percent reduction in the pain among the 3 dosage groups is reported in Table 3 and 
Table 1. Laterality of interlaminar LESI.

\begin{tabular}{|l|c|c|}
\hline Laterality of Injection & Frequency & $\begin{array}{c}\text { Percent of Total } \\
\text { (\%) }\end{array}$ \\
\hline Left & 41 & 33.3 \\
Right & 50 & 40.7 \\
Midline & 32 & 26.0 \\
\hline
\end{tabular}

Table 2. Intervertebral level of interlaminar LESI.

\begin{tabular}{|l|c|c|}
\hline Intervertebral Level & Frequency & Percent of Total (\%) \\
\hline L1-L2 & 1 & 0.8 \\
\hline L2-L3 & 4 & 3.3 \\
\hline L3-L4 & 10 & 8.1 \\
\hline L4-L5 & 63 & 51.2 \\
\hline L5-S1 & 45 & 36.6 \\
\hline
\end{tabular}

Table 3. VAS pain scores pre- and post-procedure.

\begin{tabular}{|l|c|c|}
\hline & Pre-Procedure $\dagger$ & Post-Procedure $\dagger$ \\
\hline $120 \mathrm{mg}$ & $8.89 \pm 1.32$ & $4.08 \pm 3.74$ \\
\hline $80 \mathrm{mg}$ & $9.06 \pm 1.00$ & $3.75 \pm 4.00$ \\
\hline $40 \mathrm{mg}$ & $9.00 \pm 1.00$ & $4.00 \pm 0.00$ \\
\hline
\end{tabular}

$\dagger$ Variables reported as mean \pm standard deviation

Table 4. Dosage-specific results.

\begin{tabular}{|l|c|c|c|c|}
\hline & $\mathbf{1 2 0} \mathbf{~ m g}$ & $\mathbf{8 0} \mathbf{~ m g}$ & $\mathbf{4 0} \mathbf{~ m g}$ & $\boldsymbol{P}$-value \\
\hline \# of Patients & 85 & 27 & 9 & \\
\hline Age (years) $\dagger$ & $67 \pm 14.0$ & $73 \pm 13.8$ & $73 \pm 14.0$ & $0.046^{* *}$ \\
\hline Pain Relief $(\%) \dagger$ & $57 \pm 32.1 \%$ & $51 \pm 29.7 \%$ & $51 \pm 26.1$ & 0.3347 \\
\hline Adverse Effects & 4 & 0 & 0 & \\
\hline
\end{tabular}

$\dagger$ Variables reported as mean \pm standard deviation

Fig. 1. There was no statistically significant change in the percentage reduction in the pain among the 3 dosage groups $(P=0.3347)$. The difference in the percentage change in the pain score was also not significant by gender, history of back surgery, or laterality of injection. The difference in the percentage change in the pain score was significant between L4-L5 and L5-S1 (64 \pm 26.1 vs. $45 \pm 33.5 ; P=0.021$, Fig. 2). Four patients reported adverse effects, all of whom were in the $120 \mathrm{mg}$ dosage group. These adverse effects included new-onset paresthesias, flushing of the face, and worsening pain postinjection.

There were 4 (44\%) patients in the $40 \mathrm{mg}$ group,
19 (70\%) patients in the $80 \mathrm{mg}$ group, and 57 (67\%) patients in the $120 \mathrm{mg}$ group who reported $\mathrm{a} \geq 50 \%$ pain reduction. There was not a significant difference in the proportion of patients who reported $\geq 50 \%$ pain improvement among the 3 dosage groups. The proportion of patients reported $\geq 50 \%$ pain improvement also did not differ by gender, history of back surgery, or laterality of injection.

Sixty-one out of the 123 eligible patients requested a second injection during their 2-week follow-up. Among the second injection patients, 6 (10\%) patients received $40 \mathrm{mg}, 15(25 \%)$ patients received $80 \mathrm{mg}$, and $40(66 \%)$ patients received $120 \mathrm{mg}$ of methylprednisolone in the first injection. The methylprednisolone dosage differences, if any, between the patients' respective first and second injections are broken down in Fig. 3. There was no association between whether the patients received a second injection and the patients' gender status. However, there was a significant association that was shown between whether the patients received a second injection and the history of back surgery $(P=0.0061)$. In addition, there was a marginally significant association between the patient's second injection request and the laterality of injection $(P=0.049)$, and a significant association between whether the patients received a second injection and the level of injection groups L4-L5 and L5-S1 $(P=0.0111)$.

\section{DISCUSSION}

There was no statistically significant difference in pain relief when comparing $120 \mathrm{mg}, 80 \mathrm{mg}$, and $40 \mathrm{mg}$ doses of steroid. According to these results, lower doses of methylprednisolone can have the same efficacy in pain relief with less adverse effects for the patient.

These results are in line with the published literature, in which most adverse effects experienced by patients postinjection tended to be minor. Although most major adverse effects were mainly written as case reports, one prospective study of injecting 80 mg methylprednisolone showed suppression of HPA that lasted up to 14 days before returning to normal; another prospective study compared $80 \mathrm{mg}$ to $40 \mathrm{mg}$ methylprednisolone and found that both caused sup- 


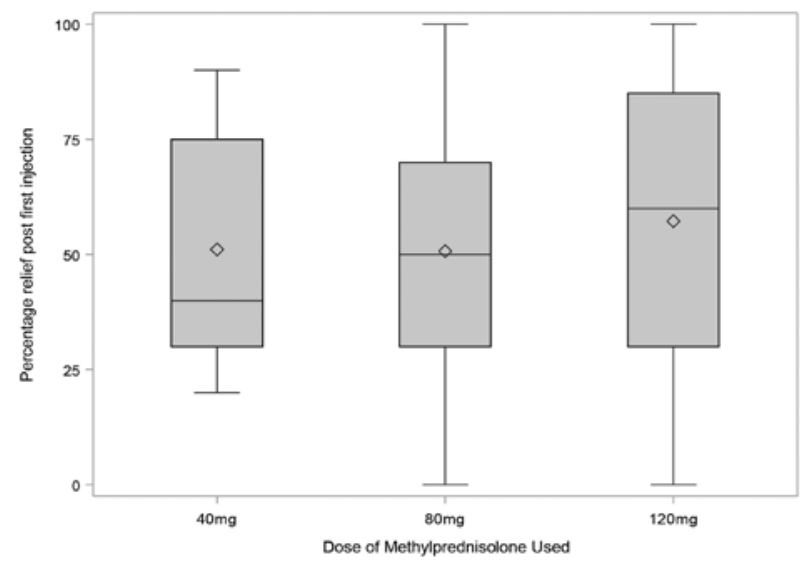

Fig. 1. Distribution of percentage pain reduction in the three dosage groups.

pression of HPA, with the $80 \mathrm{mg}$ group experiencing more clinical suppression that lasted for longer than the $40 \mathrm{mg}$ group $(11,12)$. One study looking at the pharmacokinetics of triamcinolone in patients receiving fluoroscopically guided epidural injections for chronic low-back pain reaffirmed the potential harmful effects epidural-administered steroids have on the endocrine function (13). Thus lowering the dose of methylprednisolone proves to limit not only the risk of adverse effects experienced by the patient, but also the duration of those unwanted effects as well.

Interlaminar ESI has been established in the literature as effective for pain relief in those suffering from longstanding low-back pain with radicular symptoms due to lumbar spinal stenosis or disc disease (14). A review of current literature yielded mostly low-quality data regarding the effectiveness of interlaminar ESI owing to their small sample sizes and differing diagnostic criteria; however, most studies did show statistically significant shortterm improvement in pain (15). A double-blind, randomized controlled trial of interlaminar ESI compared with saline solution injection showed statistically significant pain relief and improved function lasting approximately 3 months with interlaminar LESI, after which there was no difference between steroid and saline solution groups (16). Furthermore, another retrospective study evaluated the changes in NRS pain scores when comparing the use of $3 \mathrm{mg}$ of betamethasone versus 6

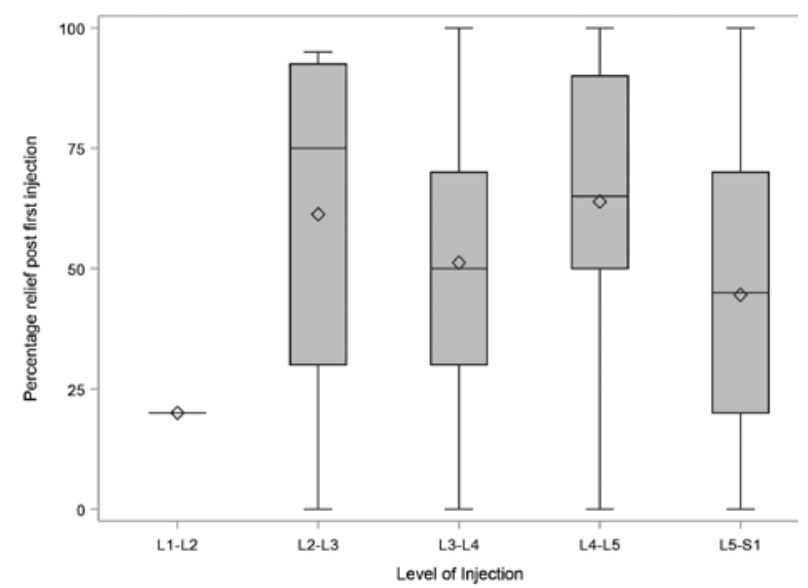

Fig. 2. Distribution of percent relief by injection level.

$\mathrm{mg}$ of betamethasone in fluoroscopically guided transforaminal ESIs and found that there were no statistically significant differences in the reduction of NRS pain scores at 4 weeks postinjection. This study also saw a statistically equivalent reduction in narcotic usage among the patients 4 weeks postinjection at follow-up (17). Although our study did not look at decreases in narcotic use between our varying methylprednisolone dosage groups, the statistically significant equivalency in preand post-injection pain scores among all dosage groups in both studies are in concordance with current ESI dosing practice guidelines.

It is important to note the limitations of the study as well. For one, the retrospective nature of the study meant only information available in the patient charts regarding pain descriptions and pre- and post-procedure pain scores could be used, and the distribution of the various doses were nonrandom. Because many patients did not return after procedures for further follow-up, the length of time these patients experienced good relief is not known. Secondly, the dosage chosen for patients is subjective and not based on any objective findings while examining the patient. Thus the amount of pain relief could potentially be different based on the various dosages, and this could have also affected the outcomes. Another important limitation is the small sample sizes in the $80 \mathrm{mg}$ and $40 \mathrm{mg}$ dosage groups compared with the $120 \mathrm{mg}$ group; therefore the findings may not reflect 


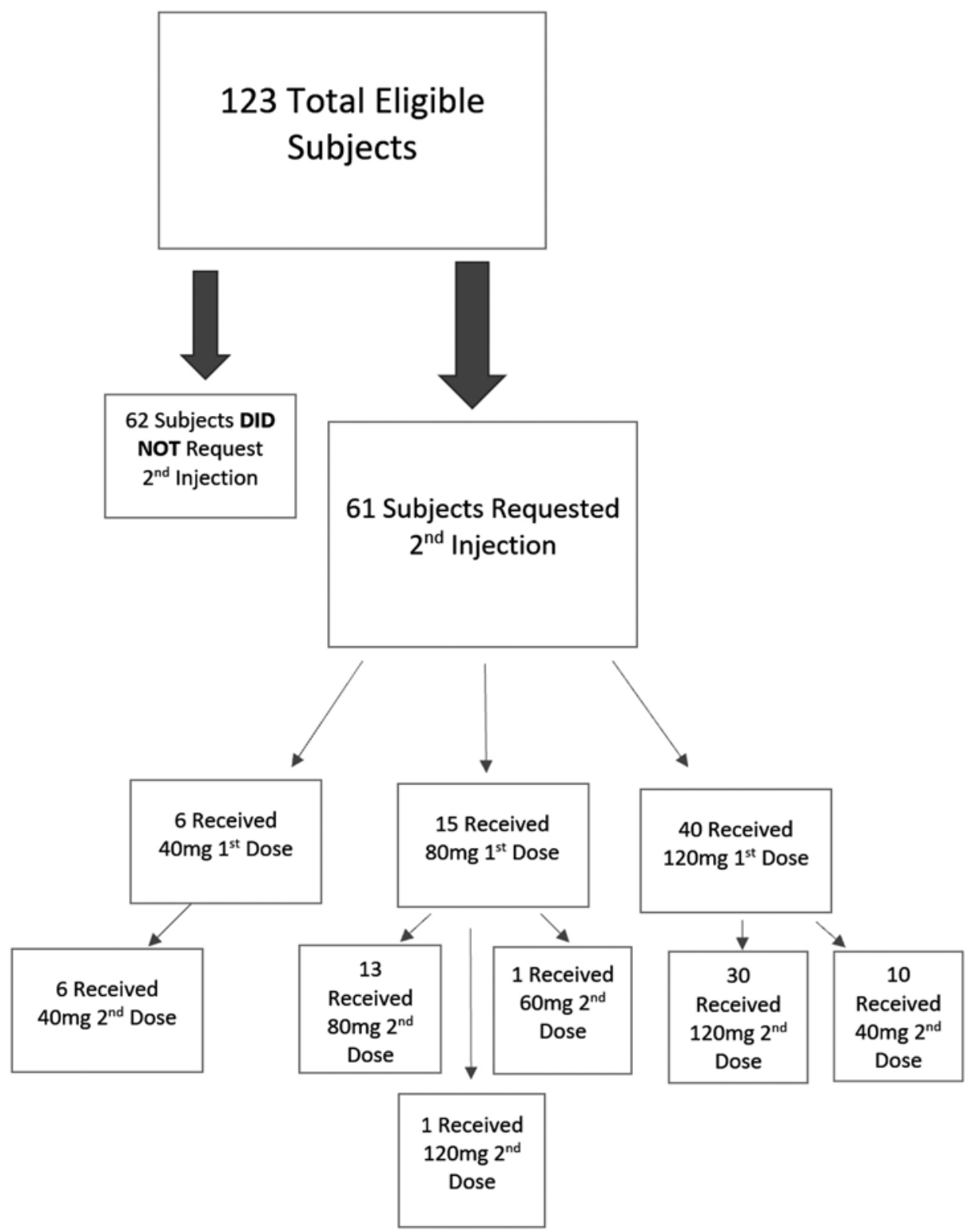

Fig. 3. Distribution of second injection methylprednisolone dosages compared to first injection methylprednisolone dosages.p

the actual effect of these dosages. In the future, a prospective trial in which patients are assigned to dosage groups in equal numbers, either through randomization or selection based on preprocedure pain scores and physical examination findings, would allow for comparing dosages with greater significance and will allow for more uniformity.

\section{CONCLUSIONS}

Our retrospective chart review demonstrates that there was no statistically significant difference in the lumbar radicular pain relief in patients who received $120 \mathrm{mg}$ of methylprednisolone and those who received the lower dosages, $80 \mathrm{mg}$ and $40 \mathrm{mg}$. This suggests that lower dosages can be used to address pain symptoms with lesser incidence of adverse ef- 
fects. Future prospective, randomized, and placebocontrolled studies are necessary to further examine the efficacy of lower dosage as treatment. Moreover, we suggest complementary studies focusing on the analgesic effect of a second low-dose methylprednisolone interlaminar LESI for those patients having $<50 \%$ pain relief after the first LESI.

\section{REFERENCES}

1. Atlas SJ, Deyo RA. Evaluating and managing acute low back pain in the primary care setting. J Gen Intern Med 2001; 16:120131.

2. Waterman BR. Low back pain in the United States: Incidence and risk factors for presentation in the emergency setting. Spine J 2012; 12:63-70.

3. Dieleman JL, Baral $\mathrm{R}$, Birger $\mathrm{M}$, et al. US spending on personal health care and public health, 1996-2013. JAMA 2016; 316:2627-2646.

4. Spijker-Huiges A, Groenhof F, Winters JC, van Wijhe M, Groenier $\mathrm{KH}$, van der Meer $\mathrm{K}$. Radiating low back pain in general practice: Incidence, prevalence, diagnosis, and long-term clinical course of illness. Scand J Prim Health Care 2015; 33:27-32.

5. Nelson DA, Landau WM. Intraspinal steroids: History, efficacy, accidentality, and controversy with review of United States Food and Drug Administration reports. J Neurol Neurosurg Psychiatry 2001; 70:433-443.

6. Cohen, SP, Bicket MC, Jamison D, Wilkinson I, Rathmell JP Epidural steroids: A comprehensive, evidence-based review. Reg Anesth Pain Med 2013; 38:175-200.

7. Collighan N, Gupta S. Epidural steroids. Contin Educ Anaesth Crit Care Pain 2010; 10:1-5.

8. Choi YK, Barbella JD. Evaluation of epidurographic contrast patterns with fluoroscopic-guided lumbar interlaminar ventral epidural injection. Pain Pract 2009; 9:275-281.

9. Byrod G, Otani K, Brisby H, Rydevik B, Olmarker K. Methylprednisolone reduces the early vascular permeability increase in spinal nerve roots induced by epidural nucleus pulposus application. J Orthop Res 2000; 18:983-987.
10. Jacobs S, Pullan PT, Potter JM, Shenfield GM. Adrenal suppression following extradural steroids. Anaesthesia 1983; 38:953956.

11. Habib G, Jabbour A, Salman J, Hakim G, Haddad H. The effect of epidural methylprednisolone acetate injection on hypopituitary-adrenal axis. J Clin Anesth 2013; 25:629-633.

12. Abdul AJ, Ghai B, Bansal D, Sachdeva N, Bhansali A, Dhatt SS. Hypothalamic pituitary adrenocortical axis suppression following a single epidural injection of methylprednisolone acetate. Pain Physician 2017; 20:E991-E1001.

13. Hooten WM, Nicholson WT, Gazelka HM, Reid JM, Moeschler SM, Lamer TJ. Serum triamcinolone levels following interlaminar epidural steroid injection. Reg Anesth Pain Med 2016; 41:75-79.

14. Okmen K, Okmen BM. The efficacy of interlaminar epidural steroid administration in multilevel intervertebral disc disease with chronic low back pain: A randomized, blinded, prospective study. Spine J 2017; 17:168-174.

15. Sharma AK, Vorobeychik $Y$, Wasserman $R$, et al. The effectiveness and risks of fluoroscopically guided lumbar interlaminar epidural steroid injections: A systematic review with comprehensive analysis of the published data. Pain Med 2017; 18:239-251.

16. Carette $\mathrm{S}$, Leclaire $\mathrm{R}$, Marcoux $\mathrm{S}$, et al. Epidural corticosteroid injections for sciatica due to herniated nucleus pulpous. $N$ Engl J Med 1997; 336:1634-1640.

17. Wong W, Maher DP, lyayi D, et al. Increased dose of betamethasone for transforaminal epidural steroid injections is not associated with superior pain outcomes at 4 weeks. Pain Physician 2015; 18:E355-E361. 
